\title{
A Scoping Review of Differential Attainment in Undergraduate Medical Education
}

\begin{abstract}
Differential attainment exists in all professions and is a manifestation of systemic factors creating an unequal environment where individual careers and aspirations may be thwarted. Although, this inequality which affects some groups of students unfairly, has been recognised over the last 2 decades, it remains a significant issue.
\end{abstract}

This scoping review explores the causes and contributors in relation to undergraduate medical education. Using thematic analysis, the authors present the case for tackling the disparity in education and training. There are evidence based solutions for individuals, organisations and at societal level. The recommendations from this review will be discussed and debated in the series of workshops, as part of the 'Bridging the Gap' series from the Alliance for Tackling Inequalities in Health, chaired by the British Association of Physicians of Indian origin. The output of the consensus building workshops and thematic synthesis with the accompanying qualitative research will be presented in the final report due in 2021.

Keywords

Differential attainment, undergraduate medicine, Bapio Institute for Health Research

\author{
Dave $\mathrm{S}^{1}$, Gupta $\mathrm{AK}^{2}$, Rishi $\mathrm{N}^{3}$, Gulati \\ $\mathrm{R}^{4}$, Varma $\mathrm{S}^{2}$, Shankar $\mathrm{R}^{5}$, Khan $\mathrm{N}^{6}$ \\ \& Chakravorty I. \\ 1. University of Bolton, UK, 2. University of \\ Birmingham, UK, 3. South London and Maudsley \\ NHS Foundation Trust, London, UK, 4. Queen's \\ University Belfast, $N$ Ireland, 5. Exeter Medical \\ School, UK, 6. Touro University Nevada, USA, 7. \\ St George's University of London, UK
}

Correspondence to:
subodh.dave@gmail.com

Cite as: Dave, S., Gupta, A.K., Rishi, N., Gulati, R., Varma, S., Shankar, R., Khan, N. \& Chakravorty, I. (2020) A Scoping Review of Differential Attainment in Undergraduate Medical Education. Sushruta J Health Pol \& Opin vol 13: issue 3: epub 1 Nov 2020 DOI: https://doi.org/10.38192/13.3.24

\section{Background}

Differential attainment (DA) is broadly defined as the variation in achievement amongst individuals based on their demographic background or attributes ${ }^{1}$, often independent of their personal effort, ability or potential. These are common due to gender, ethnicity, socio-economic status or disability and are an indicator of inequalities in society. In the context of medical education, ethnicity and international primary medical qualification stand out as ones with the largest effect sizes and impact. DA is found in both undergraduate and postgraduate training 2 and is reported in formative and summative assessments, practical skills tests and in recruitment processes. The impact of DA is pernicious; not only does it directly retard the career progression of affected professionals, but also reduces their access to senior/ more deserving roles, resulting in a deterioration of morale, higher turnover of the skilled workforce, lower retention, and subsequently poor value for money for human resources, in the NHS [3]. ${ }^{3}$

Medical school admission committees are an important gatekeeper to maintain the high standards of capability as well as the vocational aptitude of the future medical workforce, as expected by the population they are meant to serve. The admission policies and processes have a critical role in ensuring that the healthcare workforce reflect the needs of the healthcare service, therefore are subject to stricter workforce criteria by all government agencies and regulatory bodies. They are also entrusted to address some of the significant disparities that exist by geography, racial or ethnic differentials in the country. It is unfortunate, therefore, when members 
of the medical school admissions committee display significant unconscious white preference, despite acknowledging almost 'zero' explicit preferences 4. Throughout medical education including clinical placements, the opportunities to undertake electives, access research, mentorship, or targeted support remains an uneven playing field. Unconscious bias can tarnish the lived experience of students, influence decisions to pursue or avoid certain specialties, cause demoralisation and lead to isolation. Surveys amongst undergraduate and postgraduate learners often report an unacceptably high prevalence of discrimination, daily microaggressions, and sometime, being tasked as race/ethnic "ambassadors," expected to speak on behalf of their demographic groups 5 .

There is a broader ethical impact of attainment gap, for it signifies unresolved institutional issues of bias and inequality of opportunity, which undermines the principles of universality (in access to health), enshrined in the NHS Constitution ${ }^{6}$. The potential snowball detrimental impact of these systemic biases 7 need to be borne in mind, given the significant percentage of the healthcare workforce, that is either of minority ethnic origin or with primary qualification from outside the UK ${ }^{8}$. This article will focus broadly on DA in undergraduate education and specifically in medical education, and present findings from a thematic review, exploring the underlying causes and contributors.

\section{DA in Undergraduate Higher Education}

Much of the current literature on the causes of DA, and interventions targeting DA, is largely focused on postgraduate medical education 2,9. While many factors may apply equally to undergraduate and postgraduate learners, there are fundamental differences between them. Postgraduate examinations vary significantly in their structure, timing and method, which may have an influence on DA. Moreover, certain issues, isolationism, adjustment to the prevalent culture of campuses, transition away from home and economic hardship, may have a differential impact on undergraduate learners.

\section{Socio-economic factors}

Nearly $80 \%$ of all medical school admissions usually represent the most socio-economically advantaged quintile of the population. Independent school students form $<10 \%$ of all students, but attain more than a quarter of all medical school admissions ${ }^{10}$. The educational pre-attainment, parental education, house-hold income, number of members in the household, targeted support provided in high school and factors determined by the geographical and economic background of students have a significant impact on the likelihood of admission to higher ranked institutions. There is 10 a significant difference in students gaining a degree, or obtaining a graduatelevel job when comparing the most disadvantaged (fifth quintile) to the least disadvantaged socioeconomic group (first quintile).

\section{Ethnicity}

Ethnicity is another significant factor in DA. The gap between White and Black or minority ethnic student groups is widest in relation to the achievement of first or upper second-class degrees. This gap is not explained by prior attainment or performance. When comparing students with equivalent prior educational achievement (e.g. BBB grades in A-level examination) White students are significantly more likely to be awarded an upper second- or first-class degree, compared to black and minority ethnic students (70\% vs 50\%). National student surveys show that black and minority ethnic as well as students with a disability, are less likely to report receiving support and encouragement from teaching staff, have lower confidence in the fairness of assessment tools, or being satisfied with opportunities / access to learning offered by their course organisation ${ }^{11}$. Woolf et al. ${ }^{12}$ explored study habits, personality, and socio-economic background but found ethnicity emerged as an independent factor influencing DA in final examination scores, with higher scores and a greater merit or distinction rate in students of a White heritage.

\section{Gender, Sexual Orientation or Disability}

In its traditional binary sense, men and women reflect different attitudes, behaviours and skills. Gender is recognised to have an important determinant in medical education. Medical student evaluations vary depending on the gender of the student and even of the evaluator, demonstrating a gender bias in qualitative assessment of learners, obtaining letters of recommendations, where male students are described with a more positive tone and use of professional/ authoritative descriptors as compared to female residents. Fewer women are invited to speak at grand rounds and there are 'traditional' differences in the less formal introductions offered to female speakers. ${ }^{13}$

In a postal study on their own perceptions of attributes of a good doctor, there were fundamental gender-based differences. Men felt better equipped with attributes of leadership, curiosity, tolerance of uncertainty compared to the women, who felt more confident in demonstrating a caring and compassionate nature, supporting their teams, and in the ability to forge better interpersonal relationships. ${ }^{14}$ Gender also features prominently in 
stereotypical choices, medical professionals make or are encouraged to make. Throughout medical education, surgery is predominantly preferred by men and gynaecology, paediatrics and general practice by women. ${ }^{15}$

The major theoretical framework tends to be the socialization or sex-role theory, while other influencers are usually structural such as the availability of support or mentorship that women and men experience differently in making their career decisions. Women are socially programmed to expect family demands to hamper career plans, and often early negative experiences or lack of encouragement, deter women from choosing (eg. surgery) a career. Recent studies suggest that these stereotypes are shifting as lifestyle choices may influence both female and male students. ${ }^{16}$ Also, the intersectionality of gender and minority ethnic bias, often poses a double challenge for students aspiring for certain specialties. However, this definition does not include other nonracial or ethnic groups that may be underrepresented in medicine, such as lesbian, gay, bisexual, transgender, or questioning/queer or Asexual (LGBTQ+A) individuals, where data may not yet be reported. There is an estimated prevalence of $8.7 \%$ of persons with disabilities in the general population, while the prevalence of physicians with disabilities is estimated to be a mere $2.7 \% .{ }^{17}$

Stereotypes also inadvertently play a significant role in medical education. Presentation of patients and clinical vignettes often begin with a patient's age, presumed gender, and presumed racial identity. Automatic associations and mnemonics help medical students remember that, on examination, a black child with bone pain may have sickle-cell disease or a white child with recurrent respiratory infections may have cystic fibrosis. These learning associations are often based on true prevalence rates, but may not apply to individual patients, thus may lead to premature closure of the process of derivation and missed diagnoses. ${ }^{13}$

Compared with heterosexual peers, LGBTQ+A populations experience disparities in physical and mental health outcomes. Stigma and bias (both conscious and unconscious) projected by medical professionals toward the LGBTQ+A population play a major role in perpetuating these disparities and are victims of such stereotyping themselves. ${ }^{18}$ Non-white LGBTQ+A medical students experience medical school at an intersection of sexual-identity oppression and racial discrimination, often witness the unfair attitudes towards LGBTQ+A patients, feel isolated, abandon hopes of creating close relationships with educators and end up suppressing important aspects of their identities This hostile and non-inclusive environment may lead to additional stress in the work environment, contributing to poor mental health. ${ }^{192021}$

Mentorship

Optimal mentoring relationships appear to be relational, where shared values, trust, and a personal connection is important and may be more important than gender concordance. Thus students often express a desire for access to female mentors, but when a mentor and mentee develop a personal connection, the gender of the mentor becomes less important. Gender-based assumptions and stereotypes can affect mentoring relationships and influence what may be disclosed to (male) mentors and mentors in positions of power. ${ }^{22}$ One-to-one mentoring can create conditions to develop professional competences, such as reflective capacity, emotional competence and the feeling of belonging to a professional community. ${ }^{23}$

In addition to tensions of gender or sexual orientation, DA in access to mentorship can stem from issues such as power differentials or personality conflicts 24 and racial, social or cultural differences between the mentor and mentee. Often lack of role models, inexperience of the mentor, lack of motivation or institutional support may affect the access to membership for certain disadvantaged or minority groups. Teachers who share the same ethnic background as students, often demonstrate better understanding of the multi-factorial challenges, favourably judge their language proficiency in their mother tongue and perceive students as more proficient 25 than, ethnically unmatched teacher groups.

\section{Age \& Less than Full time}

Age can be a factor for determining career choices, offering hurdles, disincentives and deterrence for choices and progression. Graduate entrants to medicine are more likely to seek to immediately progress into specialty training, compared with their peers who did medicine as a primary first degree. ${ }^{26}$ Many of the IMGs, who enter postgraduate medical training in the UK are usually older than their counterparts, having spent time in postgraduate training in their home countries. There is a perception that, they therefore, are less likely to be able to adapt to the ways of the UK and take longer in acculturation. This is likely to be a determinant in their progression. Physicians with disabilities and those training in less than full time roles, often feel compelled to work twice as hard for acceptance, struggle with social 
stigma and microaggressions, and encounter institutional climates of isolation and othering. ${ }^{27}$

\section{Bias from Patients/ Service Users}

Reports of biased behaviour range from patient refusal of care and explicit racist, sexist, or homophobic remarks to belittling compliments or jokes. Targeted physicians report an emotional toll that includes exhaustion, self-doubt, and cynicism. Nontargeted bystanders report moral distress and uncertainty about how to respond. Affected individuals often respond by withdrawing from clinical roles, feel a heightened pressure in making clinical decisions, and an inexplicable fear of committing any errors. Barriers to effective functioning for students affected by such responses include lack of skills in coping with discrimination, insufficient support from senior colleagues and the institution, and perception of lack of utility associated with responding. 28

Curricula and Diversity of the Learning Environment Mountford-Zimdars at al. ${ }^{11}$ in reviewing the causes of differential outcomes in undergraduate education emphasise the importance of the learning environment noting that the learning, teaching and assessments practices of institutions are not mindful of student groups that fall outside the majority. Minority student groups do not find a 'sense of belonging' within their learning environments or indeed with the content of the curriculum and this strains relationships between staff and students or demotivates them. Due to the socio-cultural and economic capital of black and minority ethnic medical students being different from their peers, there is often a philosophical disconnect that in turn impacts their ability to network.

Woolf et al. ${ }^{29}$ report that black and minority ethnic medical students experience reduced social support and an adverse learning environment, leading to a culture of discrimination. Isik et al. 30 found that autonomous motivation was more prevalent in ethnic minorities, when comparing the motivational styles with that of the majority group in Dutch medical schools. It is likely therefore that the negative learning culture and experience that Woolf et. al. describe for black and minority ethnic students may force them away from autonomous to controlled demotivation. Similar observations have been reported from Majumder et al who describe medical education in South East Asia, as being "colonial-biased, subjectoriented, teacher-centred and hospital-based" indicating a role for a non-autonomous/ unempowering learning culture in leading to DA for certain groups. ${ }^{9}$.

In a UK General Medical Council (GMC) commissioned review de Bere et al. ${ }^{31}$ found that the causes of DA were multifactorial and embedded in educational and social factors. In order to unpick the factors explaining DA in UK medical schools, a thematic scoping review was commissioned by BAPIO Institute for Health Research (BIHR) to identify themes associated with DA. The aim of this review is to identify the mechanisms that facilitate and impede DA in UK undergraduate medical students. Identifying these themes will provide a starting point to work towards reducing inequalities in educational attainment. A brief overview of the review's methodology and key findings are presented below.

\section{Methods:}

Seven online databases including PubMed, Scopus, and ERIC were searched. A formal grey literature search was also conducted for any relevant documents through the BMA, the GMC and HEE National Grey Literature Collection. Inclusion criteria included studies dated from January 1995 to present, therefore only those pertaining to the last 25 years. Further, this review only considers UK medical students as the ethnic mix and circumstances of communities in the UK cannot be validly compared to other developed nations. All types of literature were considered, provided they were in English. Thirteen papers form the preliminary findings, selected as they provided high quality, detailed narratives on DA. The analysis has created a conceptual framework for a further mixed methods analysis. These themes have been interlinked and form the basis from which the narrative synthesis progresses.

Results

Five key themes emerged from the preliminary analysis of the thirteen papers. These themes were being 'different', social capital, continuum of discrimination, intersectionality, institutional factors, and level of external support (summarised in the table 1). 
Table 1: Themes associated with DA in undergraduate medical education

\begin{tabular}{|l|l|}
\hline Theme & Constructs \\
\hline Being Different & $\begin{array}{l}\text { Recognising individuals have different backgrounds and to be mindful } \\
\text { of these differences. }\end{array}$ \\
\hline Social Capital & $\begin{array}{l}\text { Each individual brings their own social and cultural background which } \\
\text { others may be unfamiliar with. Those in positions of responsibility for } \\
\text { teaching need to be aware of the socio-cultural differences. }\end{array}$ \\
\hline Continuum of Discrimination & $\begin{array}{l}\text { Discriminatory ideas and practices follow students throughout their } \\
\text { education and beyond, impacting their ability to aspire and their } \\
\text { confidence. }\end{array}$ \\
\hline Institutional Factors & $\begin{array}{l}\text { Institutions have not set interventions in place to tackle the DA in their } \\
\text { educational environments - partnerships between those widening } \\
\text { access and Universities need to be strengthened. }\end{array}$ \\
\hline Level of External Support & $\begin{array}{l}\text { Differential in support systems for BAME students, where they have } \\
\text { less access to help or feel less able to reach out for help - including } \\
\text { mentoring schemes. }\end{array}$ \\
\hline
\end{tabular}

\section{i: Being Different}

Black and minority ethnic undergraduate medical students in higher education, experience social isolation being of a minority culture or religion, contributing to the perception of being "different" and being 'othered'. Ethnically-defined social networks influence the informal transfer of knowledge impacting academic performance and isolating minority groups from useful academic information. The evidence that being different poses a significant disadvantage is clear - from being unable to attend social and academic functions for cultural or family reasons to feeling the pressure to change their behaviour to combat negative stereotypes in a variety of contexts, "fitting in" can be a challenge. ${ }^{32}$ In all analyses, after accounting for confounding variables, grading disparities favour White students, ${ }^{33}$ and ethnicity predicts final exam scores. 34 While subjective bias in grading, cannot be ruled out as a cause of lower grades in clinical training, 35 ethnic stereotyping of learning styles does not appear to be a major ${ }^{36,37}$ ) contributory factor. Ethnicity-related differences in clinical grades are demonstrably smaller in broadly sampled than in global assessments, however, when supervisors are allowed to deviate from original grades, ethnicity-related differences in clinical grades are reintroduced. Hence there is evidence of discrimination on the basis of ethnicity. ${ }^{38}$

The modality of assessment may lend itself to gender related disparity. Female students tend to perform better in personal and professional development, course assessment and short answer questions, while men do better in anatomy and physiology and examinations containing multiple choice or TrueFalse-Abstain (TFA) formats (Males were 17 times more likely than females to do better on an assessment, if it had any questions using the TFA format). 39

When comparing national and international students, differential item functioning analyses may demonstrate items with bias ranging from $34 \%$ to $36 \%$. While more complex items with more alternatives favoured the (predominately white) local students, shorter items and fewer alternatives favoured international students, indicating the role of linguistic ability and cultural familiarity in theoretical tests. ${ }^{40}$

\section{ii Social Capital}

Social networks provide access to a number of resources, creating channels through which resources can potentially flow between members. Connections within a close social circle, in higher education can be a significant factor in determining attainment. These networks help individuals to cope, through support and reinforcement of identity and are links between like-minded people. Cohesive groups tend to do better in medical school exams, than those outside these networks. Woolf et. al. found that students were more likely to be friends with others of the same gender and ethnicity. ${ }^{12}$ Bringing people from different social and cultural backgrounds together, helps individuals to get access to resources unavailable within their close network. Students from non-white, Muslim and lower achieving groups are least likely to gain from the benefits of social capital resulting from interaction with members from more diverse social groups. Lower levels of social capital that mediates interaction with peers, tutors and clinicians may be the cause of underperformance by ethnic minority students. Due to gaps in their social network, minority students may be cut off from 
potential and actual resources that facilitate learning and achievement. ${ }^{41}$

\section{iii- Continuum of Discrimination}

Black and minority ethnic students report feeling less satisfied with their educational experience, report anxiety and negativity in their professional interactions. This is underpinned by negative stereotypes from staff members associated with minority groups (e.g. the stereotype that Asian students are poor at communication skills). Nonnative speakers are awarded significantly lower mean scores in communication stations, while female native speakers out-perform male students. ${ }^{42}$

The use of narrative feedback has been shown to depend on gender or minority ethnic status, which suggests implicit bias. However, words that differ by gender usually refer to personal attributes, such as "lovely" in evaluations of women, while those that represent competency behaviours, such as "scientific" appear frequently in evaluations of men. It is more common for minority ethnic students being described by personal attributes, such as "pleasant" then, competency-related behaviours, such as "knowledgeable". 43 The experience of students with disability or belonging to LGBTQ+A sexual determination is no different. Some institutions recommend best practices include utilising the affirming name and pronouns for all applicants and not asking gender identity during an interview unless self-disclosed. 44

\section{iv-Support \& Mentorship}

Black and minority ethnic students are less likely to receive external support such as mentoring or sponsorship suggesting areas that universities could improve to help reduce DA. A key factor emerging from the literature is that of the repetitive, continuing nature of microaggressions. Data outlining these differentials in spheres such as postgraduate examinations or in career opportunities, serves to create a negative "continuum of discrimination" that impacts on black and minority ethnic students at multiple milestones along their career pathway.

Strategies to address DA

Our scoping review demonstrates clearly that DA is complex and exists in undergraduate medical education. The evidence from various studies also suggests measures that may help to mitigate the causes and contributors. Any strategies should include measures at individual, organisation and regional/ national level.

i. Macro level - a Culture of Inclusion

Organizations can commit to a roadmap of building capacity for change by recruitment of a critical mass of underrepresented individuals, reflecting the population that they serve and the diversity that is reflected in their workforce. Organisations should be benchmarked for the recruitment of culturally competent leaders who take the role of change agents and have the power to create diverse and equitable environments. These leaders need not themselves be from minority or underrepresented groups because culture change requires the involvement of allies within the majority groups (eg, men, white people, and cis-gender heterosexual individuals).

Committing to a culture of inclusion at the medical school level involves creating a deliberate strategy for medical student admission and evaluation and hiring, promotion, and retention of a diverse and culturally competent faculty. The Athena Swan benchmarking to encourage gender equality and recent measures to tackle the gender pay gap, had limited success. 45

ii -the power of data

Strategies for achieving diversity through medical school admissions, which includes having admissions committee members undertake robust training and reflection on potential biases, appointing women, minorities, and junior medical professionals (students or junior faculty), emphasizing the importance of different perspectives and backgrounds. There are initiatives for widening participation from socio-economically deprived communities by offering targeted support and similar incentives. Some students, however, are hampered by uncertainty as to their place within higher education, therefore need extra reassurance that their experience and beliefs are valued if they are to realise their potential. ${ }^{46}$ Collection and benchmarking of medical schools admission data on the socioeconomic profile of medical students and applicants, including characteristics such as ethnicity, gender and educational background has been achieved using the wide array of data brought together by the UK Medical Education Database, or UKMED. UKMED is run jointly by the Medical Schools Council and GMC, and in compiling the data section of the new report extensive use was made of the Higher Education Statistics Authority data within UKMED. The Selection Alliance has been using UKMED to build on these data and produce a clearer picture of the barriers faced by applicants in applying to medicine, as well as on their progress in medical school and after qualification. ${ }^{47}$ There is support from the governmental and regulatory agencies through Teaching Excellence Framework, the selection for excellence projects in encouraging medical school admissions from state school pupils, applicants with disability, but there have been many ideological counter-challenges. Applicants to higher education and students from an 'Asian or Asian British' or 'Black or Black British' background are also over-represented when compared to the UK population as measured in the 2011 UK census. Students with a declared disability 
have increased representation in medical schools from $6.6 \%$ in 2011 to $9.9 \%$ in 2015.10

Organizations should undertake, publish and implement actions from benchmarking surveys of staff perceptions of inclusivity, engagement, a sense of belonging and provide safe, responsive avenues to report experiences of bias on the grounds of cultural or demographic factors (e.g. freedom to speak up guardians). The NHS staff survey and the Workforce Race Equality standards ${ }^{48}$ have been in existence for some years. They provide a publicly available data set and national benchmarks for organisations to measure themselves against their peers. However, there is an unfortunate picture of sluggish movement towards improvement in the measures. There is transparency but lack of accountability for organisations which fail to act.

Commitment to reform from the highest leadership combined with transparency of survey data can help organizations to ensure that their training on unconscious bias and promotion of cultural humility lead to long-term positive change. Many institutions undertook a pledge to tackle discrimination and racism, with ceremonial demonstration of the phenomenon of 'taking the knee' in support of the resurgence of 'Black lives matter' movement in 2020.49 Institutions through the equality, diversity and inclusion committees, should seek and offer recommendations, from the students themselves, relate to diversity and allyship, curriculum change, open conversations, and safe spaces.

ii. Meaningful Diversity Training

Although the measures taken by the UK General Medical Council and Health Education England in training and accreditation of clinical and educational supervisors, in ensuring that equality, diversity and unconscious bias training is mandatory for all participation in education, recruitment etc, there has been little meaningful change in the markers of inequality and discrimination.

\section{iii. (Micro) Deliberative Reflection}

Before encounters that are likely to be affected by bias (such as trainee evaluations, letters of recommendation, feedback, interviews, committee decisions, and patient encounters), deliberative reflection can help an individual recognize their own potential for bias and correct for this. It is also a good time to consider the perspective of the individual whom they will be evaluating or interacting with and the potential impact of their biases on that individual. Participants can be encouraged to evaluate how their own experiences and identities influence their interactions.

This motivated self-regulation based on reflections of individual biases has been shown to reduce stereotype activation and application.

\section{iv. Strategies to address personal bias}

Individuals may question how they can actively counter stereotypes and bias in observed interactions. The active-bystander approach adapted from the Kirwan Institute can provide insight into appropriate responses in these situations.

\section{v. (Meso) Cultural Competency}

The term "cultural competence" implies that one has achieved a static goal of championing inclusivity. This approach imparts a false sense of confidence in leaders and healthcare professionals and fails to recognize that our understanding of cultural barriers is continually growing and evolving. Other synonymous terms include "cultural sensitivity" and "cultural curiosity." By training leaders and professionals that they do not need to be and ultimately cannot be experts in all the intersecting cultures that they encounter, leaders can focus on a readiness to learn that can translate to greater confidence and willingness in caring for patients of varying backgrounds. By integrating cultural humility into healthcare training procedures, organizations can strive to eliminate the perceived unease healthcare professionals might experience when interacting with individuals from backgrounds or cultures unfamiliar to them. ${ }^{50}$

\section{vi. (Meso) Counter stereotypical Interactions}

Exposing individuals to counter stereotypical experiences can have a positive impact on unconscious bias. Therefore, intentional efforts to include faculty from underrepresented groups as preceptors, educators, and invited speakers can help reduce the unconscious associations of these responsibilities as unattainable. Furthermore, in medical training, while deliberate curricula involving disparities and care of underrepresented individuals are beneficial, educators must be aware of the impact of the hidden curriculum on their trainees. The term "hidden curriculum" refers to the aspects of medicine that are learned by trainees outside the traditional classroom/didactic instruction environment. It encompasses observed interactions, behaviours, and experiences often driven by unconscious and explicit bias and institutional climate. Students can be taught to actively seek out the hidden curriculum in their training environment, reflect on the lessons, and use this reflection to inform their own behaviours.

Individuals can intentionally diversify their own circles, connecting with people from different backgrounds and experiences. This can include the occasionally awkward and uncomfortable introductions at professional meetings or at community events, making an effort to read books by diverse authors, or trying new foods with a colleague. These are small behavioural changes that, with time, 
can help to retrain our brain to classify people as "same" instead of "other."

\section{vii. Mentorship and Sponsorship}

Mentors can, at any stage in one's career, provide advice and career assistance with collaborations, but sponsors are typically more senior individuals who can curate high-profile opportunities to support a junior person, often with potential personal or professional risk if that person does not meet expectations. Possible reasons include lack of mentors from similar backgrounds or ineffective mentoring in discordant mentor-mentee relationships.

Conclusion

DA, is present in all aspects of undergraduate medical education and contributes to inequalities in higher education and impact on the healthcare workforce, as well as patient care. The mechanisms underlying DA are complex and include many causes and contributing factors from social, economic and demographic factors but also include explicit or implicit bias and processes of recruitment to assessment. There are several barriers and cultural incompetence of the majority, as well as hindered engagement from the minority, which continue to remain live issues.

Understanding the underlying mechanisms for DA is a prerequisite for designing interventions aimed at ensuring fair educational outcomes among disadvantaged students. While ethnicity may emerge as an independent risk factor for DA, gender intersectionality with factors such as socio-economic status or disability need to be considered. The current literature exploring DA within medical education has largely focused on summative assessments, particularly in the setting of final-year undergraduate exams.

Given the findings of the importance of learnereducator interactions, of the learning culture in Universities, the importance of culturally competent leadership, transparency of data, benchmarking and accountability, diverse social networks including targeted support (mentorship and sponsorship), will be important to address DA.

Our research outlines areas where interventions might be targeted. Piloting such interventions would be a natural next step. Stakeholders including medical schools, GMC and BAPIO need to be at the forefront, urgently executing these interventions to tackle DA if we are to avoid leaving behind yet another generation of doctors.

\section{References}

1. Protocol for Thematic Synthesis of Differential Attainment in the Medical Profession - 'Bridging the Gap' Series | Sushruta Journal of Health Policy \& Opinion. Accessed October 31, 2020. https://www.sushrutajnl.net/index.php/sushr uta/article/view/91

2. Dave S, Chakravorty I, Menon G, Sidhu K, Bamrah JS, Mehta R. Differential Attainment in Summative Assessments within Postgraduate Medical Education \& Training: Sushruta J Health Policy Opin. 2020;13(3). doi:10.38192/13.3.15

3. Executive summary | Falling short: the NHS workforce challenge. Health Foundation. Accessed $\quad$ October 31, 2020. https://reader.health.org.uk/fallingshort/executive-summary

4. Capers Q, Clinchot D, McDougle L, Greenwald AG. Implicit Racial Bias in Medical School Admissions. Acad Med J Assoc Am Med Coll. 2017;92(3):365-369.

\section{doi:10.1097/ACM.0000000000001388}

5. Osseo-Asare A, Balasuriya L, Huot SJ, et al. Minority Resident Physicians' Views on the Role of Race/Ethnicity in Their Training Experiences in the Workplace. JAMA Netw Open. 2018;1(5):e182723.

doi:10.1001/jamanetworkopen.2018.2723

6. The NHS Constitution for England. GOV.UK. Accessed August 31, 2020. https://www.gov.uk/government/publication s/the-nhs-constitution-for-england/the-nhsconstitution-for-england

7. Cognitive and Human Factors in Expert Decision Making: Six Fallacies and the Eight Sources of Bias | Analytical Chemistry. Accessed October 31, 2020. https://pubs.acs.org/doi/10.1021/acs.analche m.0c00704

8. Lempp H, Seale C. Medical students' perceptions in relation to ethnicity and gender: a qualitative study. BMC Med Educ. 2006;6:17. doi:10.1186/1472-6920-6-17

9. Woolf K. Fair Training Pathways for All: Understanding Experiences of Progression Final Report. :69.

10. msc-selection-alliance-2017-report.pdf.
Accessed
October
31 ,
2020. https://www.medschools.ac.uk/media/2388/ msc-selection-alliance-2017-report.pdf

11. Mountford-Zimdars A, Sanders J, Moore J, Sabri D, Jones S, Higham L. What can universities do to support all their students to progress successfully throughout their time at university? Perspect Policy Pract High Educ. 2017;21(2-3):101-110.

doi:10.1080/13603108.2016.1203368

12. Woolf K, Rich A, Viney R, Needleman S, Griffin A. Perceived causes of differential attainment in 
UK postgraduate medical training: a national qualitative study. BMJ Open. 2016;6(11):e013429. doi:10.1136/bmjopen2016-013429

13. Marcelin JR, Siraj DS, Victor R, Kotadia S, Maldonado YA. The Impact of Unconscious Bias in Healthcare: How to Recognize and Mitigate It. J Infect Dis. 2019;220(Supplement_2):S62-S73. doi:10.1093/infdis/jiz214

14. Clack GB, Head JO. Gender differences in medical graduates' assessment of their personal attributes. Med Educ. 1999;33(2):101-105. doi:10.1046/j.1365-2923.1999.00268.x

15. Alers $M$, van Leerdam L, Dielissen P, LagroJanssen A. Gendered specialities during medical education: a literature review. Perspect Med Educ. 2014;3(3):163-178. doi:10.1007/s40037-014-0132-1

16. Riska E. Gender and medical careers. Maturitas. 2011;68(3):264-267. doi:10.1016/j.maturitas.2010.09.010

17. Meeks LM, Herzer KR. Prevalence of Selfdisclosed Disability Among Medical Students in US Allopathic Medical Schools. JAMA. 2016;316(21):2271-2272. doi:10.1001/jama.2016.10544

18. Valdiserri RO, Holtgrave DR, Poteat TC, Beyrer C. Unraveling Health Disparities Among Sexual and Gender Minorities: A Commentary on the Persistent Impact of Stigma. J Homosex. 2019;66(5):571-589.

doi:10.1080/00918369.2017.1422944

19. Toman L. Navigating medical culture and LGBTQ identity. Clin Teach. 2019;16(4):335338. doi:10.1111/tct.13078

20. Sánchez NF, Rankin S, Callahan E, et al. LGBT Trainee and Health Professional Perspectives on Academic Careers--Facilitators and Challenges. LGBT Health. 2015;2(4):346-356. doi:10.1089/lgbt.2015.0024

21. Dimant OE, Cook TE, Greene RE, Radix AE. Experiences of Transgender and Gender Nonbinary Medical Students and Physicians. Transgender Health. 2019;4(1):209-216. doi:10.1089/trgh.2019.0021

22. Levine RB, Mechaber HF, Reddy ST, Cayea D, Harrison RA. "A good career choice for women": female medical students' mentoring experiences: a multi-institutional qualitative study. Acad Med J Assoc Am Med Coll. 2013;88(4):527-534. doi:10.1097/ACM.0b013e31828578bb

23. Kalén S, Ponzer S, Silén C. The core of mentorship: medical students' experiences of one-to-one mentoring in a clinical environment. Adv Health Sci Educ Theory Pract. 2012;17(3):389-401. doi:10.1007/s10459011-9317-0
24. Kow CS, Teo YH, Teo YN, et al. A systematic scoping review of ethical issues in mentoring in medical schools. BMC Med Educ. 2020;20(1):246. doi:10.1186/s12909-02002169-3

25. Glock S, Schuchart C. The ethnic match between students and teachers: evidence from a vignette study. Soc Psychol Educ. 2020;23(1):27-50. doi:10.1007/s11218-019-09525-2

26. Scanlan GM, Cleland J, Stirling SA, Walker K, Johnston P. Does initial postgraduate career intention and social demographics predict perceived career behaviour? A national crosssectional survey of UK postgraduate doctors. BMJ Open. 2019;9(8):e026444. doi:10.1136/bmjopen-2018-026444

27. Meeks LM, Herzer K, Jain NR. Removing Barriers and Facilitating Access: Increasing the Number of Physicians With Disabilities. Acad Med J Assoc Am Med Coll. 2018;93(4):540-543. doi:10.1097/ACM.0000000000002112

28. Wheeler M, de Bourmont S, Paul-Emile K, et al. Physician and Trainee Experiences With Patient Bias. JAMA Intern Med. 2019;179(12):1678. doi:10.1001/jamainternmed.2019.4122

29. Woolf K, Viney R, Rich A, Jayaweera H, Griffin A. Organisational perspectives on addressing differential attainment in postgraduate medical education: a qualitative study in the UK. $B M J$ Open. 2018;8(3). doi:10.1136/bmjopen-2017021314

30. Isik U, Wouters A, Ter Wee MM, Croiset G, Kusurkar RA. Motivation and academic performance of medical students from ethnic minorities and majority: a comparative study. BMC Med Educ. 2017;17(1):233. doi:10.1186/s12909-017-1079-9

31. Nunn S. Understanding differential attainment across medical training pathways: A rapid review of the literature. :89.

32. Claridge $\mathrm{H}$, Stone $\mathrm{K}$, Ussher $\mathrm{M}$. The ethnicity attainment gap among medical and biomedical science students: a qualitative study. BMC Med Educ. 2018;18(1):325. doi:10.1186/s12909018-1426-5

33. Low D, Pollack SW, Liao ZC, et al. Racial/Ethnic Disparities in Clinical Grading in Medical School. Teach Learn Med. 2019;31(5):487-496. doi:10.1080/10401334.2019.1597724

34. Woolf K, McManus IC, Potts HWW, Dacre J. The mediators of minority ethnic underperformance in final medical school examinations: The mediators of minority ethnic underperformance in final medical school examinations. $\mathrm{Br} J$ Educ Psychol. 2013;83(1):135-159. doi:10.1111/j.20448279.2011.02060.x

35. Stegers-Jager KM, Steyerberg EW, Cohen- 
Schotanus J, Themmen APN. Ethnic disparities in undergraduate pre-clinical and clinical performance. Med Educ. 2012;46(6):575-585. doi:10.1111/j.1365-2923.2012.04265.x

36. Stegers-Jager KM, Brommet FN, Themmen APN. Ethnic and social disparities in different types of examinations in undergraduate pre-clinical training. Adv Health Sci Educ Theory Pract. 2016;21(5):1023-1046. doi:10.1007/s10459016-9676-7

37. Stegers-Jager KM. Is it them or is it us? Unravelling ethnic disparities in undergraduate clinical performance. BMC Med. 2017;15(1):190. doi:10.1186/s12916-0170959-5

38. van Andel CEE, Born MP, Themmen APN, Stegers-Jager KM. Broadly sampled assessment reduces ethnicity-related differences in clinical grades. Med Educ. 2019;53(3):264-275. doi:10.1111/medu.13790

39. Kelly S, Dennick R. Evidence of gender bias in True-False-Abstain medical examinations. BMC Med Educ. 2009;9:32. doi:10.1186/1472-69209-32

40. Cecilio-Fernandes D, Bremers A, Collares CF, Nieuwland W, Vleuten $\mathrm{C}$ van der, Tio RA. Investigating possible causes of bias in a progress test translation: an one-edged sword. Korean J Med Educ. 2019;31(3):193-204. doi:10.3946/kjme.2019.130

41. Vaughan S, Sanders T, Crossley N, O'Neill P, Wass V. Bridging the gap: the roles of social capital and ethnicity in medical student achievement. Med Educ. 2015;49(1):114-123. doi:10.1111/medu.12597

42. Schoonheim-Klein M, Hoogstraten J, Habets L, et al. Language background and OSCE performance: a study of potential bias. Eur J Dent Educ Off J Assoc Dent Educ Eur. 2007;11(4):222-229. doi:10.1111/j.16000579.2007.00459.x

43. Rojek AE, Khanna R, Yim JWL, et al. Differences in Narrative Language in Evaluations of Medical Students by Gender and Under-represented Minority Status. J Gen Intern Med. 2019;34(5):684-691. doi:10.1007/s11606019-04889-9

44. Weinand JD, Ehlinger EP, Conniff JF, Hayon RL, Kvach E. Supporting Transgender and Nonbinary Residents. Transgender Health. 2019;4(1):222-225. doi:10.1089/trgh.2018.0074

45. Rosser SV, Barnard S, Carnes M, Munir F. Athena SWAN and ADVANCE: effectiveness and lessons learned. Lancet Lond Engl. 2019;393(10171):604-608. doi:10.1016/S0140-6736(18)33213-6

46. Garlick PB, Brown G. Widening participation in medicine. BMJ. 2008;336(7653):1111-1113. doi:10.1136/bmj.39508.606157.BE

47. Medical school widening participation work boosted by powerful data tool | Medical Schools Council. Accessed October 31, 2020. https://www.medschools.ac.uk/news/medical -school-widening-participation-work-boostedby-powerful-data-tool

48. NHS England » NHS Workforce Race Equality Standard. Accessed May 24, 2020. https://www.england.nhs.uk/about/equality/ equality-hub/equality-standard/

49. Black Lives Matter. In: Wikipedia. ; 2020. Accessed August 6, 2020. https://en.wikipedia.org/w/index.php?title=Bl ack_Lives_Matter\&oldid=971334380

50. Roberts LW. Belonging, Respectful Inclusion, and Diversity in Medical Education. Acad Med. 2020;95(5):661-664. doi:10.1097/ACM.0000000000003215

Conflict of Interest none declared

Author's Contributions

All authors contributed to the background research and writing/ editing of the manuscript. 